\title{
Evaluation of safety and efficacy of zonisamide in adult patients with partial, generalized, and combined seizures: an open labeled, noncomparative, observational Indian study
}

\author{
This article was published in the following Dove Press journal: \\ Therapeutics and Clinical Risk Management \\ 29 February 2016 \\ Number of times this article has been viewed
}

\begin{abstract}
Amitabh Dash,' Sangeeta
Ravat, ${ }^{2}$ Avathvadi Venkatesan

Srinivasan, ${ }^{3}$ Ashutosh Shetty, ${ }^{4}$

Vivek Kumar, ${ }^{5}$ Renu Achtani, ${ }^{6}$

Vivek Narain Mathur, ${ }^{7}$

Boby Varkey Maramattom, ${ }^{8}$

Veeresh Bajpai, ${ }^{9}$ Nanjappa C

Manjunath, ${ }^{10}$ Randhi Venkata

Narayana," Suyog Mehta'2

'Eisai Co. Ltd., ${ }^{2}$ Department of Neurology, Seth GS Medical College \& KEM Hospital, Mumbai, ${ }^{3}$ Department of Neurology, Trinity Acute Care Hospital, Chennai, ${ }^{4}$ Department of Neurology, Criticare Multispeciality Hospital \& Research Centre, Mumbai, ${ }^{5}$ Department of Neurology, Metro Multispeciality Hospital, Noida, ${ }^{6}$ Department of Neurology, Mata Chanan Devi Hospital, New Delhi, 'Department of Neurology, Vivekananda Hospital, Hyderabad, ${ }^{8}$ Department of Neurology, Lourdes Hospital, Kochi, ${ }^{9}$ Department of Neurology, Sai Neurology Clinic, Lucknow, ${ }^{10}$ Department of Neurology, Brain and Nerve Care, Bangalore, "Department of Neurology, Seven Hills Hospital, Visakhapatnam, ${ }^{12}$ Department of Pharmacology \& Therapeutics, Government Medical College, Solapur, India
\end{abstract}

Correspondence: Amitabh Dash Eisai Co. Ltd., Ist Floor, B Wing, Marwah Centre, Krishanlal Marwah Marg, Andheri East, Mumbai - 400072, India

Tel +9l 9l $67526 \mid 47$

Email a-dash@eisai.co.in

\begin{abstract}
A prospective, multicentric, noncomparative open-label observational study was conducted to evaluate the safety and efficacy zonisamide in Indian adult patients for the treatment of partial, generalized, or combined seizures. A total of 655 adult patients with partial, generalized, or combined seizures from 30 centers across India were recruited after initial screening. Patients received $100 \mathrm{mg}$ zonisamide as initiating dose as monotherapy/adjunctive therapy for 24 weeks, with titration of $100 \mathrm{mg}$ every 2 weeks if required. Adverse events, responder rates, and seizure freedom were observed every 4 weeks. Efficacy and safety were also assessed using Clinicians Global Assessment of Response to Therapy and Patients Global Assessment of Tolerability to Therapy, respectively. Follow-up was conducted for a period of 24 weeks after treatment initiation. A total of 655 patients were enrolled and received the treatment and 563 completed the evaluation phase. A total of $20.92 \%$ of patients received zonisamide as monotherapy or alternative monotherapy and $59.85 \%$ patients received zonisamide as first adjunctive therapy. Compared with baseline, $41.22 \%$ of patients achieved seizure freedom and $78.6 \%$ as responder rate at the end of 24 week study. Most commonly reported adverse events were loss of appetite, weight loss, sedation, and dizziness, but discontinuation due to adverse events of drug was seen in $0.92 \%$ of patients. This open label real-world study suggests that zonisamide is an effective and well-tolerated antiepileptic drug in Indian adults for treatment of partial, generalized as well as combined seizures type. No new safety signals were observed.
\end{abstract}

Keywords: zonisamide, partial onset seizures, generalized seizures, responder rate, monotherapy, adjunctive therapy

\section{Introduction}

Epilepsy is one of the most common serious neurological disorders, affecting more than 50 million people worldwide. ${ }^{1}$ It has been estimated that approximately $80 \%$ of them are in developing countries. ${ }^{2}$ The overall prevalence of epilepsy in India is reported as $1 \%$, which is slightly higher in the rural areas $(1.9 \%){ }^{2}$ Approximately twothirds of the patients experience simple or complex partial (focal) seizures, whereas the remaining one-third experience generalized seizures. ${ }^{3}$ Uncontrolled seizures can lead to a wide variety of consequences, including shortened lifespan, neuronal death, excessive bodily injury, physiological dysfunction, neuropsychological and psychiatric impairment, and social disability. ${ }^{4}$

The goal of epilepsy management currently is to maximize the quality of life of patients, by minimizing the seizure episodes, with the least possible therapy-related 
adverse effects. ${ }^{5}$ Pharmacological therapy is the most common approach for the management of epilepsy. In the past two decades, several new antiepileptic drugs (AEDs), including gabapentin, lamotrigine, levetiracetam, oxcarbazepine, topiramate, and zonisamide, have been introduced with the objective of improving efficacy, tolerability, and ease of use compared with the classical AEDs (ie, carbamazepine, valproic acid, phenytoin, phenobarbital, primidone, ethosuximide, and benzodiazepines). However, this makes the drug selection process quite challenging. Although seizures can be controlled in approximately $60 \%-70 \%$ of patients with the first or second AED, regardless of the drug used, failure to respond to the first two AEDs may suggest refractoriness. ${ }^{3}$

Zonisamide is a benzisoxazole derivative with a unique chemical structure that differs structurally from other AEDs. It is considered as one of the preferred AEDs among the newgeneration AEDs owing to its potent broad spectrum of efficacy, once-daily dosing, good tolerability, and potential for weight loss. ${ }^{5}$ Zonisamide has demonstrated safety and efficacy in a wide variety of epilepsy and seizure types, including both partial and generalized seizures, with over 2 million patient-years of exposure internationally, over the past 18 years of its existence. ${ }^{6}$ Zonisamide has International League Against Epilepsy Level A evidence as initial monotherapy for efficacy in adults with partial onset seizures. ${ }^{7}$ Furthermore, several clinical studies have reported that zonisamide is an effective and well-tolerated therapy in everyday clinical settings similar to that observed in clinical trials. ${ }^{8}$ However, all these studies were conducted in the USA and Europe and included patients with partial onset seizures with or without secondary generalization.

Taking this as background, we evaluated the efficacy and safety of zonisamide in Indian adult patients with partial, generalized, and combined seizures in real-world treatment situations and everyday clinical practice.

\section{Methods}

\section{Design and patients}

This was a prospective, multicentric, open-labeled, noncomparative, postmarketing surveillance study conducted across 30 centers in India. Patients with partial (simple partial, complex partial, and secondary generalized), generalized (tonic-clonic seizures, tonic seizures, and atypical absence seizures), or combined seizures, who satisfied all the inclusion/exclusion criteria were enrolled in the study. The study was conducted under conditions of normal clinical practice where the available data corresponding to certain specific and clinically relevant parameters were captured at regular time points (baseline, 4 weeks interval and 24 weeks). Baseline demography, physical examination, weight, vital signs, medical history, including history of patient's seizures, seizure type and epilepsy syndrome, seizure frequency, prior treatment, and AED prescription were recorded. Informed consent was obtained, and the patient's eligibility was assessed prior to the enrolment. The study was conducted in accordance with the ethical code of conduct laid out by the Declaration of Helsinki, Good Clinical Practice guidelines, and Indian Council of Medical Research guidelines. The protocol and statement of informed consent were approved by the Institutional Ethics Committee of each center. ClinicalTrials.gov identifier: NCT01283256.

The inclusion criteria were as follows: male or female subjects, aged 18-75 years, previously treated or untreated with AEDs, diagnosed with partial seizures (simple partial seizures, complex partial seizures, and secondary generalized tonicclonic seizures), generalized seizures (tonic-clonic seizures, tonic seizures, and atypical absence seizures), or combined seizures (mixed). Patients had to have computed tomography or magnetic resonance imaging done within the past 10 years that ruled out a progressive cause of epilepsy. Females of childbearing potential must have a negative pregnancy test at screening and enrollment and be willing to use medically acceptable forms of contraception for the duration of the study and for 1 month following discontinuation of the study drug.

Patients with history of seizures of nonepileptic origin, drug or alcohol abuse, kidney stones, or hypercalciuria, psychiatric disorders requiring electroconvulsive therapy in past 6 months, suicide attempt within past 6 months, or noncompliance with psychotropic medications were excluded. Those with significant past or present medical disorders, mental disorder, or any progressive neurological disorder (eg, infection, multiple sclerosis, or tumor), clinically significant metabolic acidosis, any known hypersensitivity to the study drug, zonisamide, or hypersensitivity to sulfonamides were also excluded.

Additionally, patients who were receiving zonisamide therapy, any investigational new drug, carbonic anhydrase inhibitors (acetazolamide), or mono-amine oxidase inhibitors, in the past 3 months before screening were also excluded from the study. Furthermore, addition of any other AED during the study period called for exclusion of the patient from the study.

\section{Treatment}

Patients were treated with zonisamide according to the approved package insert ${ }^{9}$ and as per the discretion of the physician. Zonisamide therapy was initiated as $100 \mathrm{mg}$ tablets once-daily after meals before bedtime, which is minimum recommended dosage for epilepsy patient aged $>18$ years 
in India. The dose was up-titrated by $100 \mathrm{mg}$ every 2 weeks till each individual patient reached the maintenance dose, depending on the dose which achieved seizure control. However, the maximum recommended and allowed dose in the study was $600 \mathrm{mg} / \mathrm{day}$.

\section{Efficacy assessments}

After the baseline-screening visit (day 0), the patients were followed-up at the end of weeks 4, 8, 12, 16, 20, and 24 (end of study visit). A window period of \pm 2 days was permitted for the follow-up visit. During each visit, a complete general examination and clinical evaluation of efficacy parameters were carried out.

The primary efficacy end-point was a median percentage reduction in seizure frequency over a period of 24 weeks. Seizure activity was recorded by the subject or relative/ caretaker in the patient diary provided, from day 1 through the end of the study. The secondary efficacy end-points were percentage responder rate and seizure-free patients over a period of 24 weeks. A subject was classified as a responder if there was $\geq 50 \%$ reduction in the frequency of seizures, regardless of seizure type, for a period of 24 weeks when on a stable dose of zonisamide. The Clinicians Global Assessment of Response to Therapy (CGART) was assessed by the clinician on a seven-point scale as compared to baseline rated as follows: $1=$ excellent response, $2=$ good response, $3=$ average response, $4=$ no response, $5=$ minimally poor response, $6=$ poor response, and $7=$ very poor response. ${ }^{10}$ In addition, the subgroup analysis of responder rate, seizure-free patients at 24 weeks, and adverse events was performed in accordance with major concomitant AEDs such as carbamazepine, valproate, phenytoin, and levetiracetam.

\section{Safety assessments}

All adverse events, either spontaneously reported by the patients, or noted by the physician were recorded during the study period. The nature and severity of the adverse effects, if any, were also recorded. Patient Global Assessment of Tolerability to Therapy (PGATT) was assessed by the patient on a four-point rating scale as follows: $1=$ excellent tolerability, $2=$ good tolerability, $3=$ average tolerability, and $4=$ poor tolerability. ${ }^{11}$ At every visit, concomitant medications received by each patient were reviewed to assess the likelihood of any adverse events.

\section{Treatment compliance}

All patients included in the study were asked to bring back the remaining medication/empty strips during the next visit and the number of tablets/empty strips was counted to check for patient compliance. If the patient had not consumed more than $80 \%$ of the total prescribed medication during every visit, then the patient was termed as "noncompliant" and was not included in the efficacy analysis.

\section{Statistical methods for data analysis}

Data from all the centers were pooled for analysis. Two populations were retained for analysis. These were the intention to treat (ITT) population, corresponding to all included patients who met the eligibility criteria, and the per protocol (PP) population, corresponding to all patients in the ITT population who completed the study.

Measurement data were expressed as mean and standard deviation, and categorical data were expressed as median and range, and nominal data were expressed as numbers and percentages. Measurement data were analyzed using one-way analysis of variance (repeat measures), whereas ranking/qualitative data were analyzed using Friedman test. Proportions of patients who were seizure-free before enrolment and those after study completion were analyzed using paired $t$-test. For all statistical tests, a $P$-value equal to 0.05 was considered significant.

\section{Results}

\section{Baseline characteristics}

Of the total 659 patients who consulted a participating neurologist, 655 patients fulfilled the criteria for inclusion in the study (ITT population). The demographic characteristics and clinical features of the study population are given in Table 1. Four patients were excluded from the study due to lack of data. Totally, 563 patients completed the 24 week study period (PP population), whereas 92 discontinued (Figure 1).

At the start of zonisamide therapy, the median seizure frequency was three seizures/month (range: 1-120). Zonisamide was administered as first-line therapy in 137 patients (20.92\%), whereas it was administered as add-on therapy in 518 patients $(79.08 \%)$. Among patients who received zonisamide as add-on therapy, approximately $59.85 \%$ of the patients received it as first add-on therapy, $16.03 \%$ as second add-on, $2.44 \%$ as third add-on, and $0.76 \%$ of patients as fourth add-on (Figure 2). The median dose of zonisamide used during the study was $200 \mathrm{mg} /$ day. Prior to starting zonisamide, 105 patients had been treated unsuccessfully with at least two AEDs, 16 with three AEDs, and five with four AEDs. The most frequently used concomitant AEDs during the study are shown in Figure 3. These include carbamazepine (25.65\% patients), valproate $(24.73 \%)$, phenytoin $(24.12 \%)$, levetiracetam $(14.05 \%)$, benzodiazepines $(12.52 \%)$, and lamotrigine (1.68\%). 
Table I Demographic and clinical features of study population

\begin{tabular}{|c|c|}
\hline & Total population $(\mathrm{N}=655)$ \\
\hline Age, mean \pm SD (years) & $33.24 \pm 13.22$ \\
\hline \multicolumn{2}{|l|}{ Sex, n (\%) } \\
\hline Male & $392(59.85 \%)$ \\
\hline Female & $263(40.15 \%)$ \\
\hline \multicolumn{2}{|l|}{ Past medical history, n (\%) } \\
\hline Epilepsy & $12(1.83)$ \\
\hline Psychiatric disorders & $0(0.00)$ \\
\hline CVA & $3(0.46)$ \\
\hline Allergy & I (0.15) \\
\hline Valproate allergy & $0(0.00)$ \\
\hline Hypertension & $21(3.21)$ \\
\hline CVD & $6(0.92)$ \\
\hline Diabetes & $12(1.83)$ \\
\hline Alcohol & $8(1.22)$ \\
\hline Smoking & $22(3.36)$ \\
\hline Hypercholesterolemia & $5(0.76)$ \\
\hline CHD & $3(0.46)$ \\
\hline \multicolumn{2}{|l|}{ Seizure type, n (\%) } \\
\hline Simple partial & $48(7.33)$ \\
\hline Complex seizures & $179(27.33)$ \\
\hline Secondary GTC & $124(18.93)$ \\
\hline Generalized TC & $200(30.53)$ \\
\hline Generalized tonic & $46(7.02)$ \\
\hline Atypical absence seizures & $4(0.61)$ \\
\hline Mixed seizures & $54(8.24)$ \\
\hline \multicolumn{2}{|c|}{ Duration of epilepsy (months), $\mathrm{n}$ (\%) } \\
\hline $0-12$ & $|7|(26.11)$ \\
\hline $12-24$ & $133(20.3 \mid)$ \\
\hline $24-36$ & $73(11.15)$ \\
\hline $36-48$ & $51(7.79)$ \\
\hline $48-60$ & $45(6.87)$ \\
\hline$>60$ & $182(27.79)$ \\
\hline \multicolumn{2}{|l|}{ Seizure features, $\mathrm{n}(\%)$} \\
\hline Pre-ictal phase & $222(33.89)$ \\
\hline Consciousness impaired & 437 (66.72) \\
\hline Motor symptoms & $417(63.66)$ \\
\hline Sensory symptoms & $163(24.89)$ \\
\hline Autonomic symptoms & $92(14.05)$ \\
\hline Psychic symptoms & $66(10.08)$ \\
\hline
\end{tabular}

Abbreviations: CHD, congestive heart disease; CVA, cerebrovascular accidents; CVD, cerebrovascular disorders; GTC, generalized tonic clonic; TC, tonic clonic; $\mathrm{SD}$, standard deviation.

\section{Efficacy}

There was a statistically significant reduction in seizure frequency at each follow-up visit compared to baseline $(P<0.0001)$, with a maximum percentage reduction of $91.5 \%$ at week $24(P<0.0001$, Figure 4$)$. The mean change in seizure frequency from baseline was -3.98 (95\% CI -3.39 to -4.57$)$. Among responders, seizure frequency was reduced from $4.52 \pm 8.13$ seizures/month at baseline to $0.21 \pm 0.78$ at 24 weeks, with mean change from baseline of -3.74 .

In the ITT population, percentage of patients who showed some significant reduction in seizure frequency was $93 \%$, whereas $7 \%$ of the patients showed no statistically significant change. In the PP population, approximately $95 \%$ of the patients showed statistical reduction in the seizure frequency and approximately $5 \%$ of the patients showed no significant change in seizure frequency.

Responder rate (defined as percentage of patients whose seizure frequency decreased by $\geq 50 \%$ compared with baseline) was $78.6 \%$; approximately $41.22 \%$ of the patients achieved seizure freedom after 24 weeks of zonisamide therapy (Figure 4).

The responder rate was not clinically different with respect to concomitant antiepileptic medication used. Responder rate was higher for Valproate $(89.51 \%)$ and lower for Phenytoin (67.09\%). However, number of patients who achieved seizure freedom was lowest for patients receiving carbamazepine (30.4\%) and highest for those receiving phenytoin (44.9\%) (Table 2).

The efficacy with zonisamide treatment assessed by the clinician on a seven-point CGART scale was "good" to "excellent" in $89.83 \%$ of the patients at week 24 (Figure 5). "Average response" was observed in 3.92\% of the patients, whereas $6.24 \%$ patients had "no response" or "poor response".

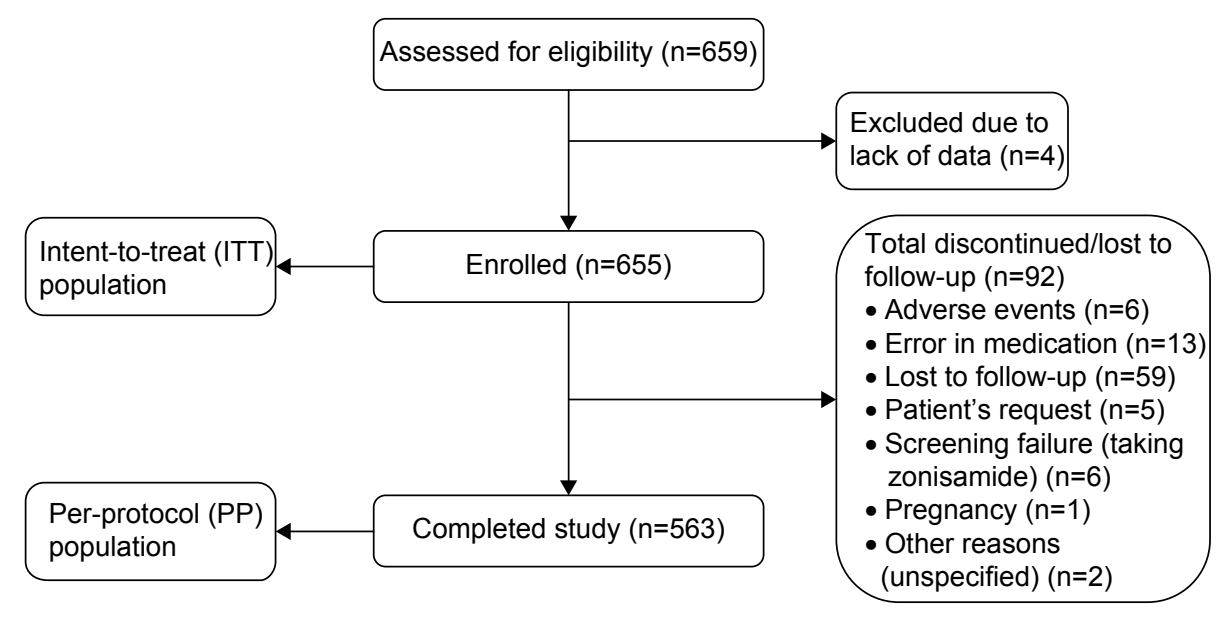

Figure I Flow diagram of the study. 


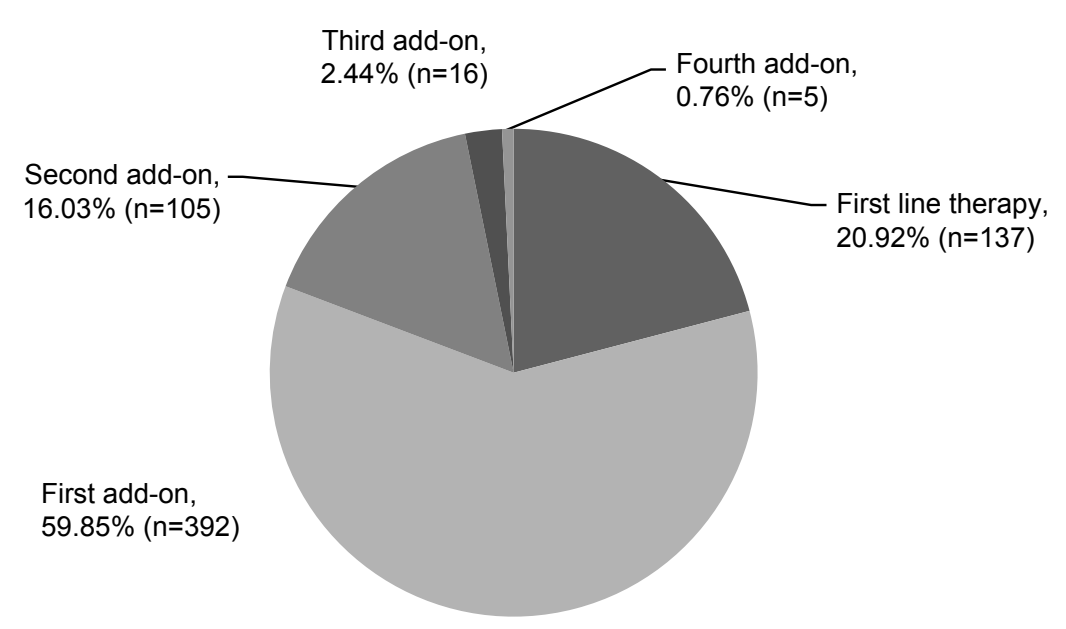

Figure 2 Type of zonisamide therapy administered.

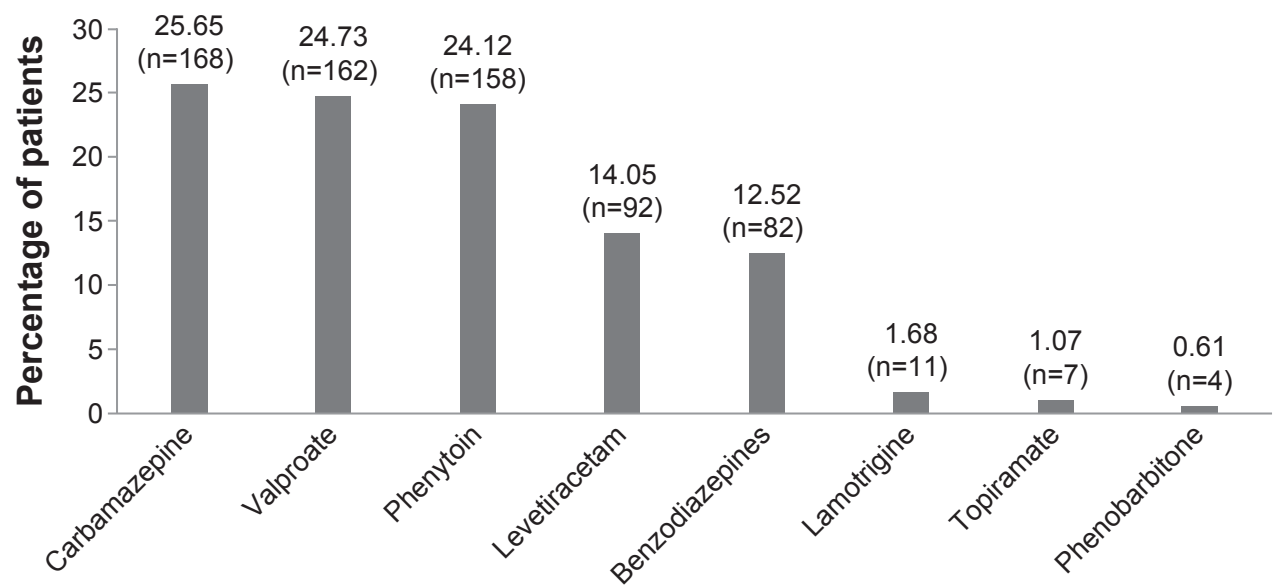

Figure 3 Concomitant antiepileptic drugs used during the study.

Notes: The data included patients who received concomitant medication to monotherapy $(n=392)$ and as combination therapy (105 patients received dual therapy, 16 patients received triple therapy, and five patients received four-drug therapy).

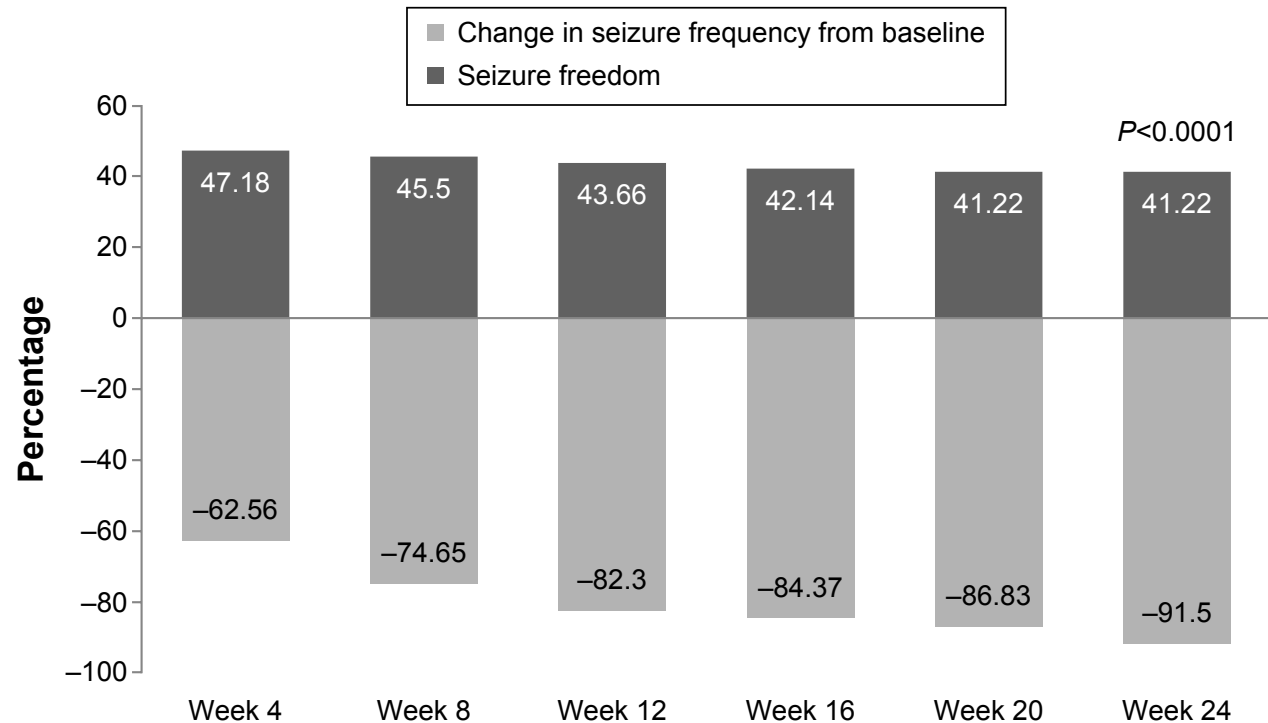

Figure 4 Percent change from baseline in seizure frequency and seizure freedom. 
Table 2 Treatment response as a function of concomitant AED

\begin{tabular}{lll}
\hline & $\begin{array}{l}\text { Seizure freedom } \\
\text { at } \mathbf{2 4} \text { weeks, \% }\end{array}$ & $\begin{array}{l}\text { Responder rate } \\
\text { at } \mathbf{2 4} \text { weeks, \% }\end{array}$ \\
\hline $\begin{array}{l}\text { Zonisamide + carbamazepine } \\
(\mathrm{n}=168)\end{array}$ & 30.4 & 69.64 \\
$\begin{array}{l}\text { Zonisamide + valproate } \\
(\mathrm{n}=162)\end{array}$ & 33.64 & $89.5 \mathrm{I}$ \\
$\begin{array}{l}\text { Zonisamide + phenytoin } \\
(\mathrm{n}=158)\end{array}$ & 44.9 & 67.01 \\
$\begin{array}{l}\text { Zonisamide + levetiracetam } \\
(\mathrm{n}=92)\end{array}$ & 35.9 & 79.35 \\
\hline
\end{tabular}

Abbreviation: AED, antiepileptic drug.

\section{Safety and tolerability}

A total of 114 adverse events (17.4\%) were reported in 112 subjects (Table 3). Majority of these events were of mildto-moderate intensity. The most commonly reported adverse events were loss of appetite (7.3\%), weight loss (3.5\%), sedation/somnolence $(2.1 \%)$, and dizziness $(2 \%)$. Increased seizure frequency was reported in three patients $(0.5 \%)$ and rashes in two patients (0.3\%). Aggressive behavior and sleep disturbance, mild rash and skin itching, fever, and headache were reported in one patient each. There was no death, disability, or hospitalization required for any of the adverse events. Furthermore, none of the events was due to over-dosage, or was congenital or malignant in nature. No history of clinical relevant metabolic acidosis or oligohydrosis was reported.

There was no incidence of kidney stone reported, possibly due to the short duration of study. Six patients $(0.92 \%)$ discontinued therapy due to adverse events. The tolerability of zonisamide assessed by patients on a four-point PGATT

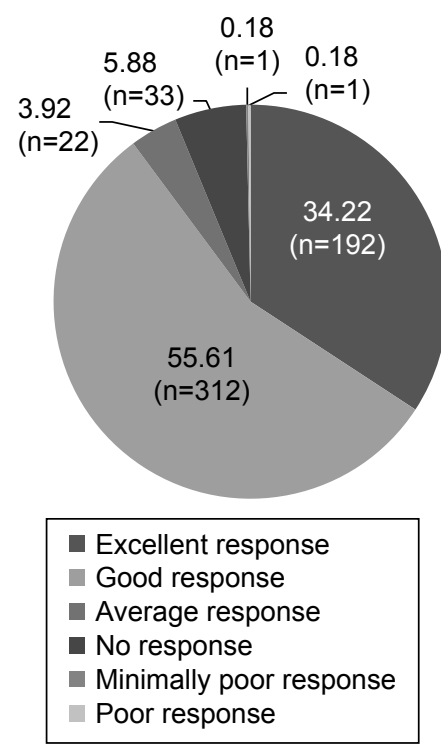

Figure 5 Clinicians Global Assessment of Response to Therapy (CGART) at week 24, $n=561$.
Table 3 Adverse events (AEs) reported during the study

\begin{tabular}{lll}
\hline $\begin{array}{l}\text { Serial } \\
\text { number }\end{array}$ & AE & Frequency, $\mathbf{n}(\%)$ \\
\hline $\mathrm{I}$ & Loss of appetite & $48(7.33)$ \\
2 & Weight loss & $23(3.5 \mathrm{I})$ \\
3 & Sedation & $14(2.14)$ \\
4 & Dizziness & $13(1.98)$ \\
5 & Irritability & $7(\mathrm{I} .07)$ \\
6 & Increased seizure frequency & $3(0.46)$ \\
7 & Rash & $2(0.3 \mathrm{I})$ \\
8 & Aggressive behavior and sleep disturbance & $\mathrm{I}(0.15)$ \\
9 & Mild rash and itching over skin & $\mathrm{I}(0.15)$ \\
I0 & Viral fever & $\mathrm{I}(0.15)$ \\
$\mathrm{II}$ & Headache & $\mathrm{I}(0.15)$ \\
& Total adverse events & $\mathrm{II}(17.40)$ \\
& Total patients & $\mathrm{II}(17.10)$ \\
\hline
\end{tabular}

scale was "good" to "excellent" in $93.75 \%$ of the patients at week 24 (Figure 6).

\section{Discussion}

This is the first observational postmarketing study comprising the largest patient pool to assess the safety and efficacy of zonisamide in Indian patients in real-world clinical settings. All other studies evaluating effects of zonisamide in realworld clinical settings were conducted mainly in Europe. In India, zonisamide has been approved as monotherapy or adjunctive therapy in patients with primary generalized seizures and partial seizures, and in Europe as monotherapy in adult patients with newly diagnosed partial seizures and adjunctive therapy in children aged $\geq 6$ years and adults with partial seizures with or without secondary generalization.

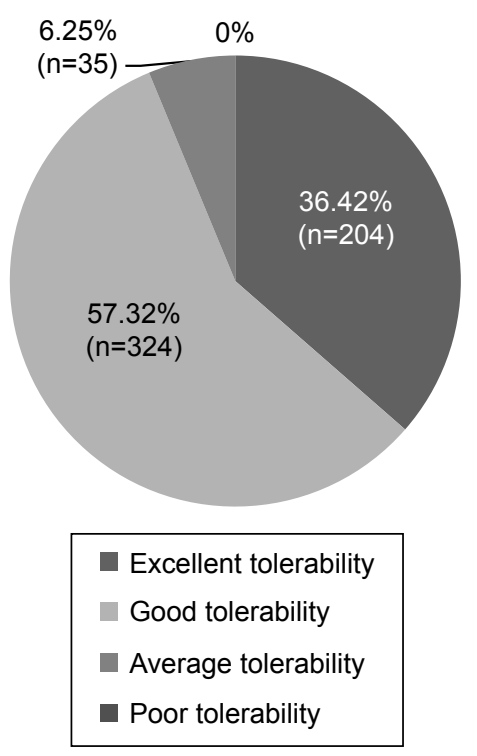

Figure 6 Patients Global Assessment of Tolerability to Therapy (PGATT) at week $24, n=560$. 
The primary outcome of this study was impressive with more than approximately $80 \%$ of patients achieving $\geq 50 \%$ reduction in seizure frequency, whereas seizure freedom was achieved in approximately $41 \%$ of patients after 24 weeks of zonisamide treatment. The responder rate and proportion of patients who achieved seizure freedom with zonisamide were $40.9 \%$ and $15 \%$, respectively, in ZEUS; ${ }^{12}$ $79 \%$ and $34 \%$ in ZADE study $;^{13} 65.9 \%$ and $25.6 \%$ in OZONE study; ${ }^{14}$ and $79.7 \%$ and $43.6 \%$ in ZOOM study. ${ }^{15}$ This difference may be attributable to inclusion of patients with less severe and less refractory epilepsies in our study. Median seizure frequency at baseline was three seizures/ month in this study, which was lower than that reported in previous randomized trials and studies conducted in real-world clinical settings. ${ }^{12-20}$ Moreover, approximately $17 \%$ of the patients in this study were not taking any AEDs while $80 \%$ were taking only one or two AEDs. Furthermore, mean duration of epilepsy in patients included in this study was approximately 5 years, which was again lower than that reported in other observational studies (approximately 23 years in ZEUS, 18 years in OZONE study, and 15 years in ZADE study). Another possible reason may be inclusion of patients with partial, generalized, and combined seizure type in comparison with previous studies which included patients with partial onset seizures.

In a Phase III multicenter randomized-controlled trial, Baulac et $\mathrm{al}^{21}$ compared efficacy and safety of zonisamide $300 \mathrm{mg} /$ day and carbamazepine $600 \mathrm{mg} /$ day monotherapy for 26-78 weeks in adults with newly diagnosed partial epilepsy. The study showed that $79.4 \%$ of the patients in PP population and $69.4 \%$ in ITT population were free of seizures at 26 weeks in the Zonisamide group, as compared to $83.7 \%$ of the patients in PP population and $74.7 \%$ in ITT population in the Carbamazepine group. These findings further support the notion that zonisamide may provide greater efficacy in newly diagnosed or less refractory patients of epilepsy.

The overall efficacy of zonisamide treatment as assessed by the clinician on a seven-point CGART scale was "good" to "excellent" in approximately $89.83 \%$ of the subjects.

With regard to safety, the study demonstrated that zonisamide was well-tolerated, with adverse events reported in approximately $17 \%$ of patients. This rate was lower than that reported in previous randomized-controlled trials and observation studies. ${ }^{12-21}$ The most common adverse events reported were decrease in appetite, weight loss, sedation, and dizziness. No cases of renal stone, metabolic acidosis, and oligohydrosis were reported. Most of the AEs were of mild-to-moderate intensity with no death, disability, or inpatient hospitalization required for any of the adverse events.
Discontinuation due to adverse effects of drug was seen in only $0.92 \%$ patients.

Despite the inherent limitations of an open label, noncomparative, and observational study design, the results of this study provide some useful clinical information. We were able to gather data across a broad age range from a relatively large group of epilepsy patients with a wide spectrum of both partial onset seizures and generalized seizures. However, long-term efficacy and safety of zonisamide could not be assessed owing to short duration of study.

\section{Conclusion}

This real-world data in clinical settings do suggest that zonisamide is an effective and well-tolerated AED for the treatment of partial, generalized, and combined seizures in the Indian population. Zonisamide is found to be effective in various seizure types which could be due to its potent broad spectrum action. ${ }^{22,23}$ The long half-life of zonisamide (63-69 hours) allows once-daily dosing, which could enhance adherence to therapy. Some AEDs such as carbamazepine, valproate, oxcarbazepine, gabapentin, and pregabalin are associated with weight gain, in contrast zonisamide is found to reduce appetite and help with weight loss. Hence, it could be advantageous in some patients when administered as combination therapy with these AEDs. ${ }^{5,24}$ However, a 24 week observational study is relatively short time to judge effectiveness of zonisamide and further long-term studies can be considered in future. The results of this study support the use of zonisamide as well-tolerated and efficacious agent for treatment of partial onset and generalized seizures. Zonisamide can be considered by physicians as a potential AED as monotherapy or adjunctive therapy to achieve seizure control in their epileptic patients in their real-world clinical settings.

\section{Acknowledgments}

This study was supported by funding from Eisai Co. Ltd. We thank all participating investigators of the study listed here: Dr Ruchir Divatia, Shrey Hospital, Ahmedabad, Gujarat; Dr Jitender Singh, Sanjivani Hospital, Ahmedabad; Dr Shivkumar, Cerebrovascular \& Vasculitis Research Foundation, Chennai; Dr Mahesh, Child Care \& Neuro Centre, T Nagar, Chennai; Dr Arulselvan, Apollo Hospital, Chennai; Dr Sreedhar, Sai Krishna super specialty Neuro Hospital, Kachiguda, Hyderabad; Dr CH Gopal, Prime Hospitals, Ameerpet, Hyderabad; Dr B Jyothi, Medplus Clinic, Vijayanagar Colony, Hyderabad; Dr Suresh Gupta, Santokba Durlabji Memorial Hospital cum Research Institute, Lucknow; Dr Sunit Kumar Shah, Advanced 
Neurology \& Super Specialty Hospital, Jaipur; Dr Neeraj Bhutani, Bhutani's NeuroCare, Jaipur; Dr Murali K Menon, Krishna Clinic, Kochi; Dr Asad Abbas, Neurocare \& Research Centre, Lucknow; Dr SS Pujari, Dr Shripad Pujari's Neurology Clinic, Pune; Dr Avanti Atul Biniwale, Biniwale Clinic, Pune; Dr Adabala Kiran, Ayush Neuro Clinic, Lothkunta, Secunderabad; Dr G Satyanarayana, Vijaya Health Care Multi Specialty Hospital, Secunderabad; Dr V Srinivas Reddy, Vijayawada Institute of Neuro Sciences Private Limited, Vijayawada; Dr N Pushpa Raju, Varun Neuro Clinic, Visakhapatnam; Dr Demudubabu, Caring Hands Neuro Center, Visakhapatnam.

\section{Disclosure}

All authors asides from Dr Amitabh Dash received an investigator fee for the study. Dr Amitabh Dash is an employee of Eisai Co. Ltd. The authors report no other conflicts of interest in this work.

\section{References}

1. Rakesh PS, Ramesh R, Rachel P, Chanda R, Satish N, Mohan VR. Quality of life among people with epilepsy: a cross-sectional study from rural southern India. Natl Med J India. 2012;25:261-264.

2. Santhosh NS, Sinha S, Satishchandra P. Epilepsy: Indian perspective. Ann Indian Acad Neurol. 2014;17(Suppl 1):S3-S11.

3. Asconapé JJ. The selection of antiepileptic drugs for the treatment of epilepsy in children and adults. Neurol Clin. 2010;28:843-852.

4. Sperling MR. The consequences of uncontrolled epilepsy. CNS Spectr. 2004;9(2):98-101, 106-109.

5. Holder JL Jr, Wilfong AA. Zonisamide in the treatment of epilepsy. Expert Opin Pharmacother. 2011;12:2573-2581.

6. Wilfong AA, Willmore LJ. Zonisamide - a review of experience and use in partial seizures. Neuropsychiatr Dis Treat. 2006;2:269-280.

7. Glauser T, Ben-Menachem E, Bourgeois B, et al. Updated ILAE evidence review of antiepileptic drug efficacy and effectiveness as initial monotherapy for epileptic seizures and syndromes. Epilepsia. 2013; 54:551-563.

8. Kwan SY, Chuang YC, Huang CW, Chen TC, Jou SB, Dash A. Zonisamide: review of recent clinical evidence for treatment of epilepsy. CNS Neurosci Ther. 2015;21(9):683-691.

9. Zonegran ${ }^{\circledR}$ (Zonisamide) [package insert]. European Knowledge Centre, Mosquito Way, Hatfield, Hertfordshire, UK; Eisai Europe Ltd; 2013.

10. Busner J1, Targum SD. The clinical global impressions scale: applying a research tool in clinical practice. Psychiatry (Edgmont). 2007; 4(7):28-37.

Therapeutics and Clinical Risk Management

\section{Publish your work in this journal}

Therapeutics and Clinical Risk Management is an international, peerreviewed journal of clinical therapeutics and risk management, focusing on concise rapid reporting of clinical studies in all therapeutic areas, outcomes, safety, and programs for the effective, safe, and sustained use of medicines. This journal is indexed on PubMed Central, CAS,
11. Prabhoo R, Panghate A, Dewda RP, More B, Prabhoo T, Rana R. Efficacy and tolerability of a fixed dose combination of methylcobalamin and pregabalin in the management of painful neuropathy. $N \mathrm{Am} \mathrm{J} \mathrm{Med}$ Sci. 2012;4(11):605-607.

12. Dupont S, Striano S, Trinka E, et al. Flexible dosing of adjunctive zonisamide in the treatment of adult partial-onset seizures: a non-comparative, open label study (ZEUS). Acta Neurol Scand. 2010;121:141-148.

13. Helmstaedter C, Stefan H, Witt JA. Quality of life in patients with partial-onset seizures under adjunctive therapy with zonisamide: results from a prospective non-interventional surveillance study. Epileptic Disord. 2011;13:263-276.

14. Dupont S, Biraben A, Lavernhe G, Marquet T, Allaf B. Management and monitoring of patients treated with zonisamide: the OZONE study. Epileptic Disord. 2013;15:278-288.

15. Hamer H, Baulac M, Bagul M, Kockelmann E. p134-retention, dosing, tolerability and patient reported seizure outcome of zonisamide as only add-on treatment under real-life conditions in adult patients with partial onset seizures: results of the non-interventional study ZOOM. Epilepsia. 2014;55(Suppl 2):4-6.

16. Schmidt D, Jacob R, Loiseau P, et al. Zonisamide for add-on treatment of refractory partial epilepsy: a European double-blind trial. Epilepsy Res. 1993;15:67-73.

17. Faught E, Ayala R, Montouris GG, Leppik IE, Zonisamide 922 trial group. Randomized controlled trial of zonisamide for the treatment of refractory partial-onset seizures. Neurology. 2001;57:1774-1779.

18. Sackellares JC, Ramsay RE, Wilder BJ, Browne TR 3rd, Shellenberger MK. Randomized, controlled clinical trial of zonisamide as adjunctive treatment for refractory partial seizures. Epilepsia. 2004;45:610-617.

19. Brodie MJ, Duncan R, Vespignani H, Solyom A, Bitenskyy V, Lucas C. Dose-dependent safety and efficacy of zonisamide: a randomized, double-blind, placebo-controlled study in patients with refractory partial seizures. Epilepsia. 2005;46:31-41.

20. Catarino CB, Bartolini E, Bell GS, Yuen AW, Duncan JS, Sander JW. The long-term retention of zonisamide in a large cohort of people with epilepsy at a tertiary referral centre. Epilepsy Res. 2011;96:39-44.

21. Baulac M, Brodie MJ, Patten A, Segieth J, Giorgi L. Efficacy and tolerability of zonisamide versus controlled release carbamazepine for newly diagnosed partial epilepsy: a phase 3, randomised, double-blind, noninferiority trial. Lancet Neurol. 2012;11:579-588.

22. Brodie MJ, Ben-Menachem E, Chouette I, Giorgi L. Zonisamide: its pharmacology, efficacy and safety in clinical trials. Acta Neurol Scand Suppl. 2012;(194):19-28.

23. Hoy SM. Zonisamide: a review of its use in the management of adults with partial seizures. Drugs. 2013;73(12):1321-1338.

24. Wellmer J, Wellmer S, Bauer J. The impact of zonisamide on weight. A clinical study in 103 patients with epilepsy. Acta Neurol Scand. 2009; 119:233-238.
EMBase, Scopus and the Elsevier Bibliographic databases. The manuscript management system is completely online and includes a very quick and fair peer-review system, which is all easy to use. Visit $\mathrm{http}: / /$ www.dovepress.com/testimonials.php to read real quotes from published authors. 